\title{
Decision Rules Acquisition for Inconsistent Disjunctive Set-Valued Ordered Decision Information Systems
}

\author{
Hongkai Wang, Yanyong Guan, Jilin Huang, and Jianting Shen \\ School of Mathematical Sciences, University of Jinan, Jinan 250022, China \\ Correspondence should be addressed to Hongkai Wang; wanghongkai@sina.com \\ Received 4 January 2015; Accepted 11 March 2015 \\ Academic Editor: Wanquan Liu \\ Copyright ( 2015 Hongkai Wang et al. This is an open access article distributed under the Creative Commons Attribution License, \\ which permits unrestricted use, distribution, and reproduction in any medium, provided the original work is properly cited.

\begin{abstract}
Set-valued information system is an important formal framework for the development of decision support systems. We focus on the decision rules acquisition for the inconsistent disjunctive set-valued ordered decision information system in this paper. In order to derive optimal decision rules for an inconsistent disjunctive set-valued ordered decision information system, we define the concept of reduct of an object. By constructing the dominance discernibility function for an object, we compute reducts of the object via utilizing Boolean reasoning techniques, and then the corresponding optimal decision rules are induced. Finally, we discuss the certain reduct of the inconsistent disjunctive set-valued ordered decision information system, which can be used to simplify all certain decision rules as much as possible.
\end{abstract}

\section{Introduction}

Rough set theory, proposed by Pawlak [1-3], has been regarded as a useful tool to conceptualize and analyze various types of data. It is applied to many fields such as machine learning, knowledge discovery, and pattern recognition. With replacement of the equivalence relation by other relations such as the tolerance relation [4-9] and the dominance relation [10-13], Pawlak rough set model has been extended to numerous generalized rough set models.

Pawlak rough set model is based on an assumption that every object in the universe of discourse is associated with some information, and objects characterized by the same information are indiscernible. It is successfully used in decision rules acquisition and attribute reduction for nominal information systems. However, Pawlak rough set model is not able to discover inconsistency for attributes in preference-ordered domains. Ordered information systems are firstly proposed and studied by Iwinski [14]. Yao and Sai $[15,16]$ transformed an ordered information table into a binary information table and then applied classical machine learning and data mining algorithms to derive ordering rules. Greco et al. [10-12] proposed the dominance-based rough set approach (DRSA), which is mainly based on substitution of the indiscernibility relation by a dominance relation, to deal with such kind of inconsistency.

The DRSA proposed by Greco et al. [10-12] mainly focuses on discussing sorting problem and extracting the dominance decision rules for a complete ordered decision information system (ODIS), in which all attribute values are exactly known. Many researchers have investigated various types of ODISs by the dominance-based rough set approach and its extended models [17-29].

Set-valued information systems are generalized models of single-valued information systems. There are many ways to give a semantic interpretation of the set-valued information systems. Guan and Wang [5] summarized them in two types: disjunctive and conjunctive systems. For set-valued ordered information systems (OISs), Qian et al. [27] defined two dominance relations in a conjunctive set-valued ODIS and a disjunctive set-valued ODIS, respectively, and used them to define lower and upper approximations. From the lower and upper approximations, certain decision rules and possible decision rules can be derived from these two types of set-valued ODISs. Furthermore, Qian et al. [28] introduced four types of dominance relations and discussed criterion reductions of disjunctive set-valued ordered information systems. However, for the reduction of the system, 
their discussions are restricted on set-valued OISs and consistent set-valued ODISs. Nevertheless, inconsistent set-valued ODISs are common in practice, so, in this paper, we will investigate the reduction of the inconsistent set-valued ODISs based on the discussion of decision rules optimization. Our discussion mainly focuses on disjunctive set-valued ODISs, and this approach is also applicable to the conjunctive setvalued ODISs.

The rest of this paper is organized as follows. In Section 2, some notations and basic concepts for the ODIS and the DRSA are introduced. In Section 3, dominance-based rough set model in disjunctive set-valued ODISs is reviewed. In Section 4, the optimization problem of decision rules in inconsistent disjunctive set-valued ODIS is discussed. The concept of reduct of an object is proposed. By constructing the dominance discernibility function for an object, reducts of an object are computed via utilizing Boolean reasoning techniques, and then the corresponding optimal decision rules are induced. In Section 5, the certain reduct of the inconsistent disjunctive set-valued ODIS, which can be used to simplify all certain decision rules as much as possible, is also discussed. Finally, we conclude our work in Section 6.

\section{The Dominance-Based Rough Set Approach}

2.1. The Ordered Decision Information System (ODIS). An information system is represented by a quadruple $S=(U$, AT, $V, f)$, where $U=\left\{x_{1}, x_{2}, \ldots, x_{m}\right\}$ is a finite nonempty set of objects, called the universe of discourse; AT is a finite set of attributes; $V=\bigcup_{a \in \mathrm{AT}} V_{a}$, where $V_{a}$ is the domain of attribute $a ; f: U \times \mathrm{AT} \rightarrow V$ is an information function satisfying $f(x, a) \in V_{a}, \forall x \in U, \forall a \in$ AT. We denote $f(x, a)=a(x)$ for simplicity. In general, if attributes in an information system are classified into condition attributes and decision attributes, then $S=(U, \mathrm{AT}, V, f)$ is called a decision information system (DIS) or called a decision table (DT). In this case, the set of condition attributes is denoted by $C=\left\{c_{1}, c_{2}, \ldots, c_{n}\right\}$, and the set of decision attributes is denoted by $D$; that is, $\mathrm{AT}=C \cup D$ with $C \cap D=\varnothing$. Without loss of generality, we assume that $D=\{d\}$ and $V_{d}=\{1,2, \ldots, r\}$. The partition of $U$ determined by $R_{d}=\{(x, y) \mid(x, y) \in U \times U, d(x)=d(y)\}$ is usually denoted as $U / R_{d}=\left\{D_{1}, D_{2}, \ldots, D_{r}\right\}$, where $D_{k}, 1 \leq k \leq r$, is called a decision class.

Further, we denote $d(X)=\{d(y) \mid y \in X\}$ for any $X \subseteq U$. Then one can conclude that $d\left(D_{k}\right)=\{k\}$.

If the domain of a condition attribute is ordered according to a decreasing or increasing preference, then the attribute is called a criterion [10-13]. Next we will introduce the concept of the ordered decision information system.

Definition 1 (see $[10,12])$. For a DIS $S=(U, \mathrm{AT}, V, f)$, if the condition attributes are criteria and the value of decision attribute $d$ represents an overall preference of objects, that is, for any $s$ and $t$ with $s>t$, the objects in $D_{s}$ are more preferred than the objects in $D_{t}$, then one calls the information system an ordered decision information system (ODIS) or ordered decision table (ODT).
In an ODIS, the domain of a criterion $a \in C$ is completely preordered by an outranking relation $>_{a}$, where $f(x, a)>_{a} f(y, a)$ implies that $x$ is at least as good as $y$ with respect to the criterion $a$. We denote such $x$ and $y$ as $x>_{a} y$ or $y \prec_{a} x$.

For $B \subseteq C$, if $B=B_{1} \cup B_{2}$ and the domain of every attribute in $B_{1}$ is ordered according to a descending preference while the domain of the attribute in $B_{2}$ is ordered according to an ascending preference, then the dominance relation determined by $B$ can be defined as

$$
\begin{aligned}
& R_{B}^{\succ}=\left\{(x, y) \mid x \succ_{a} y \forall a \in B_{1}, x \prec_{b} y \forall b \in B_{2}\right\}, \\
& R_{B}^{\prec}=\left\{(x, y) \mid x \prec_{a} y \forall a \in B_{1}, x \succ_{b} y \forall b \in B_{2}\right\} .
\end{aligned}
$$

From mathematical point of view, the ascending and descending order relations are dual, and so they can be handled similarly. Therefore, without loss of generality, we make an assumption for simplicity that values in the domain of every condition attribute are only descendingly ordered. With this assumption, we obtain

$$
\begin{aligned}
& R_{B}^{>}=\left\{(x, y) \mid x \succ_{a} y, \quad \forall a \in B\right\}, \\
& R_{B}^{\prec}=\left\{(x, y) \mid x \prec_{a} y, \quad \forall a \in B\right\} .
\end{aligned}
$$

Since $(x, y) \in R_{B}^{>}$is equivalent to $(y, x) \in R_{B}^{\prec},(x, y) \in R_{B}^{>}$ is also denoted as $x \succ_{B} y$ or $y \prec_{B} x$. From (2), we obtain that $R_{C}^{\succ} \subseteq R_{B}^{\succ}$ and $R_{C}^{\prec} \subseteq R_{B}^{\prec}$ for $B \subseteq C$.

For $B \subseteq C$, one can see that $R_{B}^{>}$is transitive, and it is also reflexive under the assumption that $x>_{a} x, \forall x \in U$, and $\forall a \in$ $C$. Furthermore, for $x, y \in U$, we denote $x={ }_{B} y$ if $b(x)=b(y)$, $\forall b \in B$, and $x \neq_{B} y$ if $\exists b \in B$ such that $b(x) \neq b(y)$. Then, $(x, y) \in R_{B}^{>}$and $(y, x) \in R_{B}^{>}$can imply $x={ }_{B} y$. Therefore, $R_{B}^{>}$ is antisymmetric. Hence, $\left(U, R_{B}^{>}\right)$is a partial ordered set [30] and so is $\left(U, R_{B}^{\prec}\right)$.

2.2. The Dominance-Based Rough Set Model. In an ODIS, for $x, y \in U$, if $(x, y) \in R_{B}^{>}$, one says that $x$ dominates $y$ or $y$ is dominated by $x$ with respect to $B$. Let us denote

$$
\begin{aligned}
& {[x]_{B}^{>}=\left\{y \mid y \in U, y \succ_{B} x\right\},} \\
& {[x]_{B}^{\prec}=\left\{y \mid y \in U, y \prec_{B} x\right\} .}
\end{aligned}
$$

Then $[x]_{B}^{>}$is called a dominating set and $[x]_{B}^{<}$a dominated set of $x$ with respect to $B$.

Further, we denote

$$
U / R_{B}^{>}=\left\{[x]_{B}^{>} \mid x \in U\right\}, \quad U / R_{B}^{\prec}=\left\{[x]_{B}^{\prec} \mid x \in U\right\} .
$$

Then, both $U / R_{B}^{\succ}$ and $U / R_{B}^{\prec}$ are coverings of $U$.

In the DRSA $[10,12,17],[x]_{B}^{>}$and $[x]_{B}^{<}(x \in U)$ are regarded as basic knowledge granules and they are used to define the lower and upper approximations as shown below.

Definition 2 (see $[10,12,17])$. In an ODIS $S=(U, C \cup d, V, f)$, for $X \subseteq U$ and $B \subseteq C$, let

$$
\begin{gathered}
\underline{R_{B}^{\succ}}(X)=\left\{x \mid x \in U,[x]_{B}^{\succ} \subseteq X\right\}, \\
\overline{R_{B}^{\succ}}(X)=\left\{x \mid x \in U,[x]_{B}^{\prec} \cap X \neq \varnothing\right\} .
\end{gathered}
$$


Then $R_{B}^{>}(X)$ is called lower approximation and $\overline{R_{B}^{\succ}}(X)$ is called upper approximation of $X$ with respect to $B$.

Analogously, one can define lower approximation $R_{B}^{<}(X)$ and upper approximation $\overline{R_{B}^{\prec}}(X)$, respectively, as

$$
\begin{gathered}
\underline{R_{B}^{<}}(X)=\left\{x \mid x \in U,[x]_{B}^{<} \subseteq X\right\}, \\
\overline{R_{B}^{<}}(X)=\left\{x \mid x \in U,[x]_{B}^{\succ} \cap X \neq \varnothing\right\} .
\end{gathered}
$$

In the DRSA, the set to be approximated is an upward union or a downward union of decision classes; that is, $C L_{s}^{>}=$ $\bigcup_{k>s} D_{k}$ or $C L_{t}^{<}=\bigcup_{k \leq t} D_{k}$ with $s, t \in V_{d}$, where $x \in C L_{s}^{>}$ implies that $x$ belongs to at least decision class $D_{s}$ and $x \in$ $C L_{t}^{<}$indicates that $x$ belongs to at most decision class $D_{t}$. By the lower and upper approximations of $C L_{s}^{>}$and $C L_{t}^{\prec}$, Greco et al. [12] proposed five types of dominance decision rules. For example, from $x \in R_{B}^{>}\left(C L_{s}^{>}\right)$, one can get an "at least" decision rule with the form "if $z>_{B} x$, then $d(z) \geq d(x)$ "; from $R_{B}^{<}\left(C L_{s}^{<}\right)$, one can get an "at most" decision rule with the form "if $z \prec_{B} x$, then $d(z) \leq d(x)$."

\section{Dominance-Based Rough Set Model in the Disjunctive Set-Valued ODIS}

3.1. Disjunctive Set-Valued Ordered Decision Information Systems. A set-valued ordered decision table (ODT) is a setvalued decision information system $S=\{U, C \cup\{d\}, V, f\}$, where $U$ is a nonempty finite set of objects; $C$ is a finite set of condition attributes; $d$ is a decision attribute with $C \cap\{d\}=\varnothing$; $V=V_{C} \cup V_{d}$, where $V_{C}$ is the set of conditional attribute values and $V_{d}$ is the set of decisional attribute values; $f$ is a mapping from $U \times C \cup\{d\}$ to $V$ such that $f: U \times C \rightarrow 2^{V_{C}}$ is a set-valued mapping and $f: U \times\{d\} \rightarrow V_{d}$ is a single-valued mapping, where $2^{V_{C}}$ is the power set of $V_{C}$.

Table 1 shows a set-valued ordered decision table.

For any $x \in U$ and $c \in C, f(x, c)$ is interpreted disjunctively. For example, if $c$ is the attribute "speaking a language" and $f(x, c)=$ \{English, French, German $\}$, then $f(x, c)$ can be interpreted as follows: $x$ speaks English, French, or German and $x$ can speak only one of them [5]. Incomplete information systems with some unknown attribute values or partial known attribute values [7-9] are such type of setvalued information system. With such consideration, we call $S=\{U, C \cup\{d\}, V, f\}$ a disjunctive set-valued ODIS.

Example 3. A disjunctive set-valued ODIS is presented in Table 1, where $U=\left\{x_{1}, x_{2}, x_{3}, x_{4}, x_{5}, x_{6}, x_{7}, x_{8}, x_{9}, x_{10}\right\}, C=$ $\left\{c_{1}, c_{2}, c_{3}, c_{4}\right\}, V_{C}=\{0,1,2\}$, and $V_{d}=\{1,2,3\}$.

Qian et al. [28] gave four possible dominance relations in disjunctive set-valued ODISs as follows.

Definition 4 (see [28]). Let $S=(U, C \cup\{d\}, V, f)$ be a disjunctive set-valued ODIS, and $B \subseteq C$. The following four possible dominance relations between objects are considered.
TABLE 1: A disjunctive set-valued ODIS $S=(U, C \cup\{d\}, V, f)$.

\begin{tabular}{cccccc}
\hline$U$ & $c_{1}$ & $c_{2}$ & $c_{3}$ & $c_{4}$ & $d$ \\
\hline$x_{1}$ & $\{0,1\}$ & $\{1,2\}$ & $\{2\}$ & $\{1,2\}$ & 1 \\
$x_{2}$ & $\{0\}$ & $\{0,1\}$ & $\{1\}$ & $\{1\}$ & 2 \\
$x_{3}$ & $\{2\}$ & $\{2\}$ & $\{0,1\}$ & $\{1\}$ & 2 \\
$x_{4}$ & $\{0,1\}$ & $\{0\}$ & $\{0\}$ & $\{1\}$ & 1 \\
$x_{5}$ & $\{2\}$ & $\{1\}$ & $\{0,1\}$ & $\{0,1\}$ & 2 \\
$x_{6}$ & $\{1,2\}$ & $\{0\}$ & $\{0\}$ & $\{0,1\}$ & 2 \\
$x_{7}$ & $\{1\}$ & $\{1,2\}$ & $\{1,2\}$ & $\{2\}$ & 3 \\
$x_{8}$ & $\{1,2\}$ & $\{2\}$ & $\{1\}$ & $\{1,2\}$ & 3 \\
$x_{9}$ & $\{0,1\}$ & $\{0\}$ & $\{1,2\}$ & $\{0\}$ & 2 \\
$x_{10}$ & $\{2\}$ & $\{1,2\}$ & $\{1\}$ & $\{1,2\}$ & 3 \\
\hline
\end{tabular}

(I) Up dominance relation:

$$
\begin{aligned}
& R_{B}^{U \geq} \\
& =\{(y, x) \in U \times U \mid \forall a \in B, \max f(y, a) \geq \max f(x, a)\} .
\end{aligned}
$$

If $(y, x) \in R_{B}^{U \geq}$, we say that $y$ is at least up-good as $x$ with respect to $B$.

(II) Down dominance relation:

$$
\begin{aligned}
& R_{B}^{D \geq} \\
& =\{(y, x) \in U \times U \mid \forall a \in B, \min f(y, a) \geq \min f(x, a)\} .
\end{aligned}
$$

If $(y, x) \in R_{B}^{D \geq}$, we say that $y$ is at least down-good as $x$ with respect to $B$.

(III) Up-down dominance relation:

$$
\begin{aligned}
& R_{B}^{U D \geq} \\
& =\{(y, x) \in U \times U \mid \forall a \in B, \max f(y, a) \geq \min f(x, a)\} .
\end{aligned}
$$

If $(y, x) \in R_{B}^{U D \geq}$, we say that $y$ is at least possible-good as $x$ with respect to $B$.

(IV) Down-up dominance relation:

$$
\begin{aligned}
& R_{B}^{D U \geq} \\
& =\{(y, x) \in U \times U \mid \forall a \in B, \min f(y, a) \geq \max f(x, a)\} .
\end{aligned}
$$

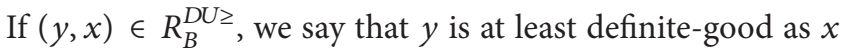
with respect to $B$.

Let $S=(U, C \cup\{d\}, V, f)$ be a disjunctive set-valued ODIS, and $R_{\{d\}}^{\geq}=\{(x, y) \mid d(x) \geq d(y)\}$. If $R_{C}^{\Delta \geq} \subseteq R_{\{d\}}^{\geq}$, then $S$ is called $\Delta$-consistent; otherwise it is called $\Delta$-inconsistent, where $\Delta$ stands for $U, D, U D$, or $D U$, respectively. 
For example, Table 1 is a $U$-inconsistent disjunctive set-valued ordered decision information system, because $R_{C}^{U \geq} \subseteq R_{\{d\}}^{\geq}$ does not hold.

Furthermore, Qian et al. [28] also gave four definitions of dominating set and dominated set of the four possible dominance relations between objects in disjunctive setvalued ODIS, respectively.

Let $[x]_{A}^{\Delta \geq}=\left\{y \in U \mid(y, x) \in R_{A}^{\Delta \geq}\right\}$ and $[x]_{A}^{\Delta \leq}=\{y \in U \mid$ $\left.(x, y) \in R_{A}^{\Delta \geq}\right\}$, where $\Delta$ equals $U, D, U D$, or $D U$, respectively. Then, $[x]_{A}^{\Delta \geq}$ consists of objects that $\Delta$-dominate $x$ and $[x]_{A}^{\Delta \leq}$ consists of objects $\Delta$-dominated by $x$.

Example 5. Compute the dominating sets induced by up dominance relation in Table 1 . We have $U / R_{C}^{U \geq}=\left\{\left[x_{1}\right]_{C}^{U \geq}\right.$, $\left.\left[x_{2}\right]_{C}^{U \geq}, \ldots,\left[x_{10}\right]_{C}^{U \geq}\right\}$, where

$$
\begin{aligned}
& {\left[x_{1}\right]_{C}^{U \geq}=\left\{x_{1}, x_{7}\right\},} \\
& {\left[x_{2}\right]_{C}^{U \geq}=\left\{x_{1}, x_{2}, x_{3}, x_{5}, x_{7}, x_{8}, x_{10}\right\},} \\
& {\left[x_{3}\right]_{C}^{U \geq}=\left\{x_{3}, x_{8}, x_{10}\right\},} \\
& {\left[x_{4}\right]_{C}^{U \geq}=\left\{x_{1}, x_{3}, x_{4}, x_{5}, x_{6}, x_{7}, x_{8}, x_{10}\right\},} \\
& {\left[x_{5}\right]_{C}^{U \geq}=\left\{x_{3}, x_{5}, x_{8}, x_{10}\right\},} \\
& {\left[x_{6}\right]_{C}^{U \geq}=\left\{x_{3}, x_{5}, x_{6}, x_{8}, x_{10}\right\},} \\
& {\left[x_{7}\right]_{C}^{U \geq}=\left\{x_{1}, x_{7}\right\},} \\
& {\left[x_{8}\right]_{C}^{U \geq}=\left\{x_{8}, x_{10}\right\},} \\
& {\left[x_{9}\right]_{C}^{U \geq}=\left\{x_{1}, x_{7}, x_{9}\right\},} \\
& {\left[x_{10}\right]_{C}^{U \geq}=\left\{x_{8}, x_{10}\right\} .}
\end{aligned}
$$

3.2. Lower and Upper Approximations in the Disjunctive SetValued ODIS. The decision attribute $d$ makes a partition of $U$ into a finite number of classes. Let $D=\left\{D_{1}, D_{2}, \ldots, D_{r}\right\}$ be a set of these classes which are ordered; that is, for any $i, j \leq r$, if $i \geq j$, then the objects in $D_{i}$ are preferred to the objects in $D_{j}$.

The sets to be approximated are an upward union and a downward union of classes, which are denoted by $\bigcup_{k \geq s} D_{k}$ $(1 \leq s \leq r)$ and $\bigcup_{k \leq t} D_{k}(1 \leq t \leq r)$, respectively. The statement $x \in \bigcup_{k>s} D_{k}$ means that " $x$ belongs to at least class $D_{s}$," whereas $x \in \bigcup_{k \leq t} D_{k}$ means that " $x$ belongs to at most class $D_{t}$ "

Analogous to the idea of decision approximation $[12,25]$, Qian et al. [28] also gave the definitions of the lower and upper approximations of $\bigcup_{k \geq s} D_{k}(1 \leq s \leq r)$ with respect to the dominance relation $R_{B}^{\Delta \geq}$ ( $\Delta$ stands for $U, D, U D$, or $D U$, resp.) in a disjunctive set-valued ODIS.

Definition 6 (see [28]). Let $S=(U, C \cup\{d\}, V, f)$ be a disjunctive set-valued ODIS, $B \subseteq C$, and $D=\left\{D_{1}, D_{2}, \ldots, D_{r}\right\}$ is the set of decision classes induced by $d$; the lower and upper approximations of $\bigcup_{k \geq s} D_{k}(1 \leq s \leq r)$ with respect to the dominance relation $R_{B}^{\Delta \geq}$ are defined as

$$
\begin{aligned}
& \underline{R_{B}^{\Delta \geq}}\left(\bigcup_{k \geq s} D_{k}\right)=\left\{x \in U \mid[x]_{B}^{\Delta \geq} \subseteq \bigcup_{k \geq s} D_{k}\right\}, \\
& \overline{R_{B}^{\Delta \geq}}\left(\bigcup_{k \geq s} D_{k}\right)=\bigcup_{x \in \bigcup_{k \geq s} D_{k}}[x]_{B}^{\Delta \geq} .
\end{aligned}
$$

The $B$-boundaries of $\bigcup_{k \geq s} D_{k}(1 \leq s \leq r)$ can be defined as

$$
B n_{B}\left(\bigcup_{k \geq s} D_{k}\right)=\overline{R_{B}^{\Delta \geq}}\left(\bigcup_{k \geq s} D_{k}\right)-\underline{R_{B}^{\Delta \geq}}\left(\bigcup_{k \geq s} D_{k}\right) .
$$

Similarly, the lower and upper approximations and boundaries of $\bigcup_{k \leq t} D_{k}(1 \leq t \leq r)$ with respect to the corresponding dominance relation in a disjunctive set-valued ODIS can also be defined.

Without loss of generality, next we will focus on the up dominance relation $R_{B}^{U \geq}$ in inconsistent disjunctive setvalued ODIS.

3.3. Decision Rules Derived from Inconsistent Disjunctive SetValued ODIS. It is noteworthy that, in the DRSA for inducing "at least" decision rules or "at most" decision rules, we need to compute the lower and upper approximations. Similarly, from the lower approximation of $\bigcup_{k \geq s} D_{k}$ ( or $\bigcup_{k \leq t} D_{k}$ ), we can induce the "at least" (or "at most") decision rules from inconsistent disjunctive set-valued ODISs. In detail, we can derive the certain "at least" decisions from the lower approximation of $\bigcup_{k \geq s} D_{k}$ and the possible "at least" decisions from the boundary of $\bigcup_{k \geq s} D_{k}$ as shown below.

(1) For $B \subseteq C$, from $x_{i} \in R_{B}^{U \geq}\left(\bigcup_{k \geq s} D_{k}\right)$, we can obtain a certain $D_{B}^{U \geq}$-decision rule:

$$
\text { if } x \in\left[x_{i}\right]_{B}^{U \geq} \text {, then } x \in \bigcup_{k \geq s} D_{k} \text { certainly holds. }
$$

It can also be equivalently stated as

if $x>_{B} x_{i}$, then $d(x) \geq s$ certainly holds, or

if $\max f\left(x, b_{1}\right) \geq \max q_{i 1}$ and $\max f\left(x, b_{2}\right) \geq \max q_{i 2}$ and $\ldots$ and $\max f\left(x, b_{l}\right) \geq \max q_{i l}$, then $d(x) \geq s$ certainly holds, where $B=\left\{b_{1}, b_{2}, \ldots, b_{l}\right\}, f\left(x_{i}, B\right)=$ $\left(q_{i 1}, q_{i 2}, \ldots, q_{i l}\right)$

Particularly, from $x_{i} \in R_{B}^{U \geq}\left(D_{k}\right)$ with $k \in V_{d}$, we can obtain a definite decision rule:

if $x>_{B} x_{i}$, then $d(x)=k$ certainly holds.

(2) For $B \subseteq C$, from $x_{i} \in B n_{B}\left(\bigcup_{k \geq s} D_{k}\right)$, we can obtain a possible $D_{B}^{U \geq}$-decision rule:

if $x \in\left[x_{i}\right]_{B}^{U \geq}$, then $x \in \bigcup_{k \geq s} D_{k}$ possibly holds, or equivalently

if $x>_{B} x_{i}$, then $d(x) \geq s$ possibly holds, or equivalently 
if $\max f\left(x, b_{1}\right) \geq \max q_{i 1}$ and $\max f\left(x, b_{2}\right) \geq \max q_{i 2}$ and ... and $\max f\left(x, b_{l}\right) \geq q_{i l}$, then $d(x) \geq s$ possibly holds.

Analogously, we can derive the certain "at most" decision rules from the lower approximation of $\bigcup_{k \leq t} D_{k}$ and the possible "at most" decision rules from the boundary of $\bigcup_{k \leq t} D_{k}$ in inconsistent disjunctive set-valued ODISs.

In order to conveniently discuss the optimization problem for the decision rules in inconsistent disjunctive setvalued ODIS, we will give another definition for the decision rules in the following.

Definition 7. In an inconsistent disjunctive set-valued ODIS $S=(S, C \cup\{d\}, V, f)$, for $B \subseteq C$ and $x_{i} \in R_{B}^{U \geq}\left(\bigcup_{k \geq s} D_{k}\right)$ with $s, t \in V_{d}, 1 \leq s \leq t \leq r$, let

$$
t_{B}^{U \geq}\left([x]_{B}^{U \geq}\right)=\bigwedge_{b \in B}(\max b, \geq, \max b(x)),
$$

where $\bigwedge$ is the conjunctive operator. We call the Boolean expression $t_{B}^{U \geq}\left([x]_{B}^{U \geq}\right)$ a condition attribute descriptor or simply $B^{U \geq}$-descriptor determined by $[x]_{B}^{U \geq}$. Then, the $D_{B}^{U \geq \text { - }}$ decision rule generated by $x_{i} \in R_{B}^{U \geq}\left(\bigcup_{k \geq s} D_{k}\right)$ can be presented as in the following form: $\bigwedge_{b \in B}(\max b, \geq, \max b(x)) \rightarrow$ $d \geq s$, where $\max b(x)$ is the maximum element in $b(x)$.

For example, in the inconsistent disjunctive set-vlaued ODIS presented in Table $1, C^{U \geq}$-descriptor determined by $\left[x_{3}\right]_{C}^{U \geq}$ is

$$
\begin{aligned}
t_{B}^{U \geq}\left(\left[x_{3}\right]_{B}^{U \geq}\right)= & \bigwedge_{b \in C}\left(\max b, \geq, \max b\left(x_{3}\right)\right) \\
= & \left(\max c_{1}, \geq, 2\right) \wedge\left(\max c_{2}, \geq, 2\right) \\
& \wedge\left(\max c_{3}, \geq, 1\right) \wedge\left(\max c_{4}, \geq, 1\right) .
\end{aligned}
$$

And the decision rule generated by $x_{3} \in R_{C}^{U \geq}\left(\bigcup_{k \geq 2} D_{k}\right)$ can be represented as

$$
\begin{aligned}
& \left(\max c_{1}, \geq, 2\right) \wedge\left(\max c_{2}, \geq, 2\right) \wedge\left(\max c_{3}, \geq, 1\right) \\
& \wedge\left(\max c_{4}, \geq, 1\right) \longrightarrow d \geq 2
\end{aligned}
$$

Without loss of generality, next we will focus on drawing the optimal "at least" decision rules in inconsistent disjunctive set-valued decision information system.

\section{The Optimization of the Decision Rules in an Inconsistent Disjunctive Set-Valued ODIS}

4.1. The Reducts of an Object and the Optimal Decision Rules. In a disjunctive set-valued ODIS $S=(U, C \cup\{d\}, V, f)$, to optimize the decision rule $\bigwedge_{b \in C}(\max b, \geq, \max b(x)) \rightarrow$ $d \geq s$ generated by $x \in \underline{R_{C}^{U \geq}}\left(\bigcup_{k \geq s} D_{k}\right)$, one should simplify as much as possible its condition part by deleting some conjunctive terms and meanwhile keep its decision part unchanged.
After deleting some terms from $\bigwedge_{b \in C}(\max b, \geq$, $\max b(x))$, we could obtain a descriptor $\bigwedge_{b \in B}(\max b, \geq$, $\max b(x))$ with $B \subseteq C$. Generally speaking, $[x]_{B}^{U \geq} \supseteq[x]_{C}^{U \geq}$ holds for any $B \subseteq C$. If $B$ satisfies $[x]_{B}^{U \geq} \supset[x]_{C}^{U \geq}$, then $d\left([x]_{B}^{U \geq}\right) \supset d\left([x]_{C}^{U \geq}\right)$ may hold, and this may lead to $x \notin R_{B}^{U \geq}\left(\bigcup_{k \geq s} D_{k}\right)$; that is, the decision value of at least one object $x \in\left[x_{i}\right]_{B}^{U \geq}$ may not satisfy $d(x) \geq s$. Therefore, to optimize the decision rule generated by $x \in R_{B}^{U \geq}\left(\bigcup_{k \geq s} D_{k}\right)$, one should delete some conjunctive terms from $\bigwedge_{b \in C}(\max b, \geq, \max b(x))$ as much as possible with the constraint condition that the set consisting of condition attributes in remainder $\bigwedge_{b \in C}(\max b, \geq, \max b(x))$ should satisfy $\min \left\{d\left([x]_{B}^{U \geq}\right)\right\} \geq s$.

Based on the above discussions, we can see that the optimization of the decision rule generated by $x \in$ $R_{C}^{U \geq}\left(\bigcup_{k \geq s} D_{k}\right)$ depends on seeking $B, B \subseteq C$, which satisfies $\overline{\min }\left\{d\left([x]_{B}^{U \geq}\right)\right\} \geq s$. So, we give the following definition for reduct of an object.

Definition 8. Let $x \in \underline{R_{C}^{U \geq}}\left(\bigcup_{k \geq s} D_{k}\right)$ and $B \subseteq C$. If $B$ satisfies $\min \left\{d\left([x]_{B}^{U \geq}\right)\right\} \geq s$, then one calls $B$ a dominance consistent set of $x$; if $B$ is a minimal subset of $C$ satisfying $\min \left\{d\left([x]_{B}^{U \geq}\right)\right\} \geq s$, then one calls $B$ a reduct of $x$. The set of all reducts of $x$ is denoted as $\operatorname{red}(x)$.

Definition 9. Let $x \in R_{C}^{U \geq}\left(\bigcup_{k \geq s} D_{k}\right)$. If $B$ is a dominance consistent set of $x$, then one calls $\bigwedge_{b \in B}(\max b, \geq$, $\max b(x)) \rightarrow d \geq s$ a simplified decision rule of $\bigwedge_{b \in C}(\max b, \geq \max b(x)) \rightarrow d \geq s$. If $B$ is a reduct of $x$, then one calls $\bigwedge_{b \in B}(\max b, \geq, \max b(x)) \rightarrow d \geq s$ an optimal decision rule of $\bigwedge_{b \in C}(\max b, \geq, \max b(x)) \rightarrow d \geq s$.

It is obvious that, with all reducts of an object, one can induce all the optimal decision rules associated with this object. Next we will discuss how to compute the reducts of an object.

\subsection{The Computation of the Reducts of an Object. For $x, y \in$} $U$, let $\alpha^{U \succ}(x, y)=\{b \mid b \in C, \max b(x)<\max b(y)\}$.

Obviously, $\alpha^{U \succ}(x, y)$ is a set of condition attributes which discern $x$ from the dominating set of $y$ or equivalently discern $y$ from the dominated set of $x$.

One can observe that $x \in[y]_{C}^{U \geq} \Leftrightarrow y \in[x]_{C}^{U \leq} \Leftrightarrow$ $\alpha^{U \succ}(x, y)=\varnothing$ or equivalently $x \notin[y]_{C}^{U \geq} \Leftrightarrow y \notin[x]_{C}^{U \leq} \Leftrightarrow$ $\alpha^{U \succ}(x, y) \neq \varnothing$.

In order to compute the reducts of an object, we will construct the discernibility function for an object. For this purpose, we give the following Theorem.

Theorem 10. For $x \in R_{C}\left(\bigcup_{k \geq s} D_{k}\right)$ and $B \subseteq C$, one has $\min \left\{d\left([x]_{B}^{U \geq}\right)\right\} \geq s \Leftrightarrow \overline{\alpha^{U} \succ}(y, x) \cap B \neq \varnothing$ for any $y$ with $d(y)<s$.

Proof. " $\Rightarrow$ " Assume that there exists $y \in U$ such that $d(y)<$ $s$, and it satisfies $\alpha^{U \succ}(y, x) \cap B \neq \varnothing$. Then, $\forall b \in B$, 
$b \notin \alpha^{U \succ}(y, x)$. From the definition of $\alpha^{U \succ}(y, x)$, we have $\max b(y) \geq \max b(x), \forall b \in B$; that is, $y \in[x]_{B}^{U \geq}$. So, by the assumption that $\min \left\{d\left([x]_{B}^{U \geq}\right)\right\} \geq s$, we can derive $d(y) \geq s$, which is contradictive to $d(y)<s$. This indicates that $\alpha^{U \succ}(y, x) \cap B \neq \varnothing$ must be satisfied by any $y$ such that $d(y)<s$.

“ $\Leftarrow$ " Assume that $\min \left\{d\left([x]_{B}^{U \geq}\right)\right\} \geq s$ does not hold, and then $\exists y \in[x]_{B}^{U \geq}$ such that $d(y)<s$. From the condition assumption, we can derive that $\alpha^{U \succ}(y, x) \cap B \neq \varnothing$. Hence, there exists at least one attribute $b \in B$ satisfying $b \in$ $\alpha^{U \succ}(y, x)$; equivalently $y \notin[x]_{B}^{U \geq}$. This is contradictive to $y \in[x]_{B}^{U \geq}$.

This completes the proof.

Based on Theorem 10, we can construct the dominance discernibility function defined below, which can help us to compute the reducts of an object.

Definition 11. For $x \in R_{C}^{U \geq}\left(\bigcup_{s \leq k} D_{k}\right)$, let

$$
\Delta^{U \geq}(x)=\bigwedge_{d(y)<s}\left(\bigvee \alpha^{U>}(y, x)\right),
$$

where $\Lambda$ is the conjunctive operator, $\bigvee$ is the disjunctive operator, and $\bigvee \alpha^{U \succ}(x, y)$ is the disjunction of all members of $\alpha^{U \succ}(x, y)$. One calls $\Delta^{U \geq}(x)$ a dominance discernibility function of $x$.

Based on Definition 11 and Theorem 10, we can obtain the following by the Boolean reasoning techniques $[3,17,31]$.

Proposition 12. For $x \in R_{C}^{U \geq}\left(\bigcup_{k \geq s} D_{k}\right)$ and $B \subseteq C$, one has that $B$ is a reduct of $[x]_{C}^{U \geq}$ if and only if $\wedge B$ is a prime implicant of $\Delta^{U \geq}(x)$, where $\bigwedge B=\bigwedge_{b \in B} b$.

Proposition 12 gives a computational method for the reducts of an object using the dominance discernibility function. Next we will give an illustrative example.

Example 13. Continuation of Example 3: we will compute all reducts of $x_{3} \in R_{C}^{U \geq}\left(\bigcup_{k \geq 2} D_{k}\right)$ and $x_{8} \in R_{C}^{U \geq}\left(\bigcup_{k \geq 3} D_{k}\right)$ and obtain those corresponding optimal decision rules.

From $\left[x_{3}\right]_{C}^{U \geq}=\left\{x_{3}, x_{8}, x_{10}\right\}$ and $\{y \mid d(y)<2\}=\left\{x_{1}, x_{4}\right\}$, we have

$$
\begin{aligned}
\Delta^{U \geq}\left(x_{3}\right) & =\left(\bigvee \alpha^{U \succ}\left(x_{1}, x_{3}\right)\right) \wedge\left(\bigvee \alpha^{U \succ}\left(x_{4}, x_{3}\right)\right) \\
& =c_{1} \wedge\left(c_{1} \vee c_{2} \vee c_{3}\right)=c_{1} .
\end{aligned}
$$

From Proposition 12, $\left\{c_{1}\right\}$ is a unique reduct of the object $x_{3}$, and it will generate the optimal decision rule of decision rule (18) induced by $x_{3}$ as follows:

$$
\left(\max c_{1}, \geq, 2\right) \longrightarrow d \geq 2 .
$$

Taking $x_{8} \in R_{C}^{U \geq}\left(\bigcup_{k \geq 3} D_{k}\right)$ as another example, we can derive the following decision rule:

$$
\begin{aligned}
& \left(\max c_{1}, \geq, 2\right) \wedge\left(\max c_{2}, \geq, 2\right) \wedge\left(\max c_{3}, \geq, 1\right) \\
& \wedge\left(\max c_{4}, \geq, 2\right) \longrightarrow d \geq 3 .
\end{aligned}
$$

From $\left[x_{8}\right]_{C}^{U \geq}=\left\{x_{8}, x_{10}\right\}$ and $\{y \mid d(y)<3\}=\left\{x_{1}, x_{2}, x_{3}\right.$, $\left.x_{4}, x_{5}, x_{6}, x_{9}\right\}$, we have

$$
\begin{aligned}
\Delta^{U \geq}\left(x_{8}\right)= & \left(\bigvee \alpha^{U \succ}\left(x_{1}, x_{8}\right)\right) \wedge\left(\bigvee \alpha^{U \succ}\left(x_{2}, x_{8}\right)\right) \\
& \wedge\left(\bigvee \alpha^{U \succ}\left(x_{3}, x_{8}\right)\right) \wedge\left(\bigvee \alpha^{U \succ}\left(x_{4}, x_{8}\right)\right) \\
& \wedge\left(\bigvee \alpha^{U \succ}\left(x_{5}, x_{8}\right)\right) \wedge\left(\bigvee \alpha^{U>}\left(x_{6}, x_{8}\right)\right) \\
& \wedge\left(\bigvee \alpha^{U \succ}\left(x_{9}, x_{8}\right)\right) \\
= & c_{1} \wedge\left(c_{1} \vee c_{2} \vee c_{4}\right) \wedge c_{4} \wedge\left(c_{1} \vee c_{2} \vee c_{3} \vee c_{4}\right) \\
& \wedge\left(c_{2} \vee c_{4}\right) \wedge\left(c_{2} \vee c_{3} \vee c_{4}\right) \wedge\left(c_{1} \vee c_{2} \vee c_{4}\right) \\
= & c_{1} \wedge c_{4} .
\end{aligned}
$$

Then one can see that $x_{8}$ has one reduct $\left\{c_{1}, c_{4}\right\}$, which generates the optimal rule of rule (22) as follows:

$$
\left(\max c_{1}, \geq, 2\right) \wedge\left(\max c_{2}, \geq, 2\right) \longrightarrow d \geq 3 .
$$

In the same way, we can compute the dominance discernibility functions of all other objects and induce their corresponding optimal decision rules.

\section{The Certain Reduct of an Inconsistent Disjunctive Set-Valued ODIS}

Based on the above discussion of reduction of the objects, in this section, we will discuss the reduct of the inconsistent disjunctive set-valued ODIS.

Definition 14. In an inconsistent disjunctive set-valued ODIS $S=(U, C \cup\{d\}, V, f), U / R\{d\}=\left\{D_{1}, D_{2}, \ldots, D_{r}\right\}$. If $B$ is a minimal subset of $C$ satisfying $R_{B}^{U \geq}\left(\bigcup_{k \geq s} D_{k}\right)=$ $R_{C}^{U \geq}\left(\bigcup_{k \geq s} D_{k}\right), 1 \leq s \leq r$, one calls $B$ a certain reduct of the inconsistent disjunctive set-valued ODIS. The intersection of all certain reducts is called certain core of the information system.

By Definitions 8, 9, and 14, it can be seen that a certain reduct of the inconsistent disjunctive set-valued ODIS is a minimal subset of condition attributes which can be used to simplify all certain decision rules as much as possible.

By Definitions 11 and 14, we can construct the dominance discernibility function of certain reduct of the inconsistent disjunctive set-valued ODIS as follows.

Definition 15. Let

$$
\Delta^{U \geq}=\bigwedge_{1 \leq s \leq r} \bigwedge_{x \in R_{C}^{U \geq}\left(\bigcup_{k \geq s} D_{k}\right)} \bigwedge_{d(y)<s} \bigvee\left(\alpha^{U>}(y, x)\right),
$$


and one calls $\Delta^{U \geq}$ dominance discernibility function of certain reduct in the inconsistent disjunctive set-valued ODIS.

Based on Definitions 11, 14, and 15 and Theorem 10, we obtain the folowing by the Boolean reasoning techniques.

Proposition 16. $B$ is a certain reduct of the inconsistent disjunctive set-valued ODIS if and only if $\wedge B$ is a prime implicant of $\Delta^{U \geq}$, where $\bigwedge B=\bigwedge_{b \in B} b$.

Proposition 16 gives a computational method for the certain reducts of the disjunctive set-valued ODIS. And we can get all corresponding simplified certain decision rules.

Example 17. Continuation of Example 3: by Definition 6, we have

$$
\begin{aligned}
& \underline{R_{C}^{U \geq}}\left(\bigcup_{k \geq 1} D_{k}\right)=\left\{x_{1}, x_{2}, x_{3}, x_{4}, x_{5}, x_{6}, x_{7}, x_{8}, x_{9}, x_{10}\right\} \\
& \underline{R_{C}^{U \geq}}\left(\bigcup_{k \geq 2} D_{k}\right)=\left\{x_{3}, x_{5}, x_{6}, x_{8}, x_{10}\right\} ; \\
& \underline{R_{C}^{U \geq}}\left(\bigcup_{k \geq 3} D_{k}\right)=\left\{x_{8}, x_{10}\right\} \\
& \Delta^{U \geq}=\bigwedge_{1 \leq s \leq 3} \bigwedge_{x \in \underline{R_{C}^{U \geq}}\left(\bigcup_{k \geq s} D_{k}\right)} \bigwedge_{d(y)<s}\left(\bigvee \alpha^{U>}(y, x)\right) \\
& =\left(\bigwedge_{x \in \underline{R_{C}^{U \geq}}\left(\bigcup_{k \geq 1} D_{k}\right)} \bigwedge_{d(y)<1}\left(\bigvee \alpha^{U>}(y, x)\right)\right) \\
& \wedge\left(\bigwedge_{x \in \underline{R_{C}^{U \geq}}} \bigwedge_{\left(\bigcup_{k \geq 2} D_{k}\right)} \bigwedge_{d(y)<2}\left(\bigvee \alpha^{U>}(y, x)\right)\right) \\
& \wedge\left(\bigwedge_{x \in R_{C}^{U \geq}\left(\bigcup_{k \geq 3} D_{k}\right)} \bigwedge_{d(y)<3}\left(\bigvee \alpha^{U>}(y, x)\right)\right) \\
& =\left(\bigwedge_{x \in \underline{R_{C}^{U \geq}}\left(\bigcup_{k \geq 2} D_{k}\right)} \bigwedge_{d(y)<2}\left(\bigvee \alpha^{U>}(y, x)\right)\right) \\
& \wedge\left(\bigwedge_{x \in R_{C}^{U \geq}\left(\bigcup_{k \geq 3} D_{k}\right)} \bigwedge_{d(y)<3}\left(\bigvee \alpha^{U>}(y, x)\right)\right) \\
& =c_{1} \wedge\left(c_{1} \wedge c_{4}\right) \\
& =c_{1} \wedge c_{4} \text {. }
\end{aligned}
$$

We can see that the considered inconsistent disjunctive set-valued ODIS presented in Table 1 has one certain reduct $\left\{c_{1}, c_{4}\right\}$.
From the lower approximations of three upward unions of decision classes, we can draw the following certain decision rules from the disjunctive set-valued ODIS presented in Table 1:

$$
\begin{aligned}
& \left(\max c_{1}, \geq, 2\right) \wedge\left(\max c_{2}, \geq, 0\right) \wedge\left(\max c_{3}, \geq, 0\right) \\
& \wedge\left(\max c_{4}, \geq, 1\right) \longrightarrow d \geq 2 \\
& \left(\max c_{1}, \geq, 2\right) \wedge\left(\max c_{2}, \geq, 2\right) \wedge\left(\max c_{3}, \geq, 1\right) \\
& \wedge\left(\max c_{4}, \geq, 2\right) \longrightarrow d \geq 3 .
\end{aligned}
$$

The certain reduct of the system, $\left\{c_{1}, c_{4}\right\}$, can be used to simplify the above two certain rules (27) as follows:

$$
\begin{aligned}
& \left(\max c_{1}, \geq, 2\right) \wedge\left(\max c_{4}, \geq, 1\right) \longrightarrow d \geq 2 ; \\
& \left(\max c_{1}, \geq, 2\right) \wedge\left(\max c_{4}, \geq, 2\right) \longrightarrow d \geq 3 .
\end{aligned}
$$

Generally speaking, a certain reduct of an inconsistent disjunctive set-valued ODIS must be a dominance consistent set of all objects from the lower approximations of the upward unions of decision classes. And a certain reduct of an inconsistent disjunctive set-valued ODIS may be a reduct of some objects from the lower approximations of the upward unions of decision classes. In Example 17, the certain reduct $\left\{c_{1}, c_{4}\right\}$ is a reduct of the object $x_{8}$, while the certain reduct $\left\{c_{1}, c_{4}\right\}$ is only a dominance consistent set of the object $x_{3}$.

\section{Conclusions}

In this paper, optimization problem of decision rules in inconsistent disjunctive set-valued ordered decision information system has been investigated. By constructing the dominance discernibility function for an object, reducts of the object are computed via utilizing Boolean reasoning techniques, and the corresponding optimal decision rules are induced. In addition, we have also discussed the certain reduct of the inconsistent disjunctive set-valued ordered decision information system and give the computational approaches using Boolean reasoning techniques.

It is well known that consistent disjunctive set-valued ordered decision information system can be seen as a special case of inconsistent disjunctive set-valued ordered decision information system. So, the approaches proposed in this paper can be used to induce optimal decision rules in the consistent disjunctive set-valued ordered decision information system.

\section{Conflict of Interests}

The authors declare that there is no conflict of interests regarding the publication of this paper.

\section{Acknowledgments}

This research is supported by a grant from the National Natural Science Foundation of China (61070241) and a grant from the Shandong Provincial Natural Science Foundation, China (ZR2013AQ007). 


\section{References}

[1] Z. Pawlak, "Rough sets," International Journal of Computer \& Information Sciences, vol. 11, no. 5, pp. 341-356, 1982.

[2] Z. Pawlak, Rough Sets: Theoretical Aspects of Reasoning about Data, System Theory, Kluwer Academic Publishers, Boston, Mass, USA, 1991.

[3] Z. Pawlak and A. Skowron, "Rough sets and Boolean reasoning," Information Sciences, vol. 177, no. 1, pp. 41-73, 2007.

[4] E. A. Abo-Tabl, "A comparison of two kinds of definitions of rough approximations based on a similarity relation," Information Sciences, vol. 181, no. 12, pp. 2587-2596, 2011.

[5] Y.-Y. Guan and H.-K. Wang, "Set-valued information systems," Information Sciences, vol. 176, no. 17, pp. 2507-2525, 2006.

[6] Y.-Y. Guan, H.-K. Wang, Y. Wang, and F. Yang, "Attribute reduction and optimal decision rules acquisition for continuous valued information systems," Information Sciences, vol. 179, no. 17, pp. 2974-2984, 2009.

[7] M. Kryszkiewicz, "Rough set approach to incomplete information systems," Information Sciences, vol. 112, no. 1-4, pp. 39-49, 1998.

[8] M. Kryszkiewicz, "Rules in incomplete information systems," Information Sciences, vol. 113, no. 3-4, pp. 271-292, 1999.

[9] Y. Leung and D. Li, "Maximal consistent block technique for rule acquisition in incomplete information systems," Information Sciences, vol. 153, pp. 85-106, 2003.

[10] S. Greco, B. Matarazzo, and R. Slowinski, "A new rough set approach to multicriteria and multiattribute classification," in Rough Sets and Current Trends in Computing, vol. 1424 of Lecture Notes in Computer Science, pp. 60-67, Springer, Berlin, Germany, 1998.

[11] S. Greco, B. Matarazzo, and R. Slowinski, "Rough sets theory for multicriteria decision analysis," European Journal of Operational Research, vol. 129, no. 1, pp. 1-47, 2001.

[12] S. Greco, B. Matarazzo, and R. Slowinski, "Rough approximation by dominance relations," International Journal of Intelligent Systems, vol. 17, no. 2, pp. 153-171, 2002.

[13] S. Greco, B. Matarazzo, R. Slowinski, and J. Stefanowski, "Variable consistency model of dominance-based rough sets approach," in Rough Sets and Current Trends in Computing, W. Ziarko and Y. Yao, Eds., vol. 2005 of Lecture Notes in Computer Science, pp. 170-181, Springer, Berlin, Germany, 2001.

[14] T. B. Iwinski, "Ordinal information system," Bulletin of the Polish Academy of Sciences Mathematics, vol. 430, no. 36, pp. 467-475, 1988.

[15] Y. Sai, Y. Y. Yao, and N. Zhong, "Data analysis and mining in ordered information tables," in Proceedings of the 1st IEEE International Conference on Data Mining (ICDM '01), pp. 497504, IEEE, San Jose, Calif, USA, December 2001.

[16] Y. Y. Yao, B. Zhou, and Y. H. Chen, "Interpreting low and high order rules: a granular computing approach," in Proceedings of the International Conference on Rough Sets and Emerging Intelligent System Paradigms (RSEISP '07), vol. 4585 of Lecture Notes in Computer Science, pp. 371-380, 2007.

[17] M. W. Shao and W. X. Zhang, "Dominance relation and rules in an incomplete ordered information system," International Journal of Intelligent Systems, vol. 20, no. 1, pp. 13-27, 2005.

[18] X. B. Yang, J. Y. Yang, C. Wu, and D. J. Yu, "Dominance-based rough set approach and knowledge reductions in incomplete ordered information system," Information Sciences, vol. 178, no. 4, pp. 1219-1234, 2008.
[19] Y. H. Qian, J. Y. Liang, and C. Y. Dang, "Interval ordered information systems," Computers and Mathematics with Applications, vol. 56, no. 8, pp. 1994-2009, 2008.

[20] X. B. Yang, D. J. Yu, J. T. Yang, and L. H. Wei, “Dominance-based rough set approach to incomplete interval-valued information system," Data \& Knowledge Engineering, vol. 68, no. 11, pp. 13311347, 2009.

[21] C. Luo, T. Li, and H. Chen, "Dynamic maintenance of approximations in set-valued ordered decision systems under the attribute generalization," Information Sciences, vol. 257, pp. 210228, 2014.

[22] Q. H. Hu, M. Z. Guo, D. R. Yu, and J. F. Liu, "Information entropy for ordinal classification," Science in China, Series F: Information Sciences, vol. 53, no. 6, pp. 1188-1200, 2010.

[23] M. Inuiguchi, Y. Yoshioka, and Y. Kusunoki, "Variable-precision dominance-based rough set approach and attribute reduction," International Journal of Approximate Reasoning, vol. 50, no. 8, pp. 1199-1214, 2009.

[24] W. Kotłowski, K. Dembczyński, S. Greco, and R. Słowiński, "Stochastic dominance-based rough set model for ordinal classification," Information Sciences, vol. 178, no. 21, pp. 40194037, 2008.

[25] W.-H. Xu, X.-Y. Zhang, J.-M. Zhong, and W.-X. Zhang, "Attribute reduction in ordered information systems based on evidence theory," Knowledge and Information Systems, vol. 25, no. 1, pp. 169-184, 2010.

[26] Y. Kusunoki and M. Inuiguchi, "A unified approach to reducts in dominance-based rough set approach," Soft Computing, vol. 14, no. 5, pp. 507-515, 2010.

[27] Y. H. Qian, C. Y. Dang, J. Y. Liang, and D. Tang, "Set-valued ordered information systems," Information Sciences, vol. 179, no. 16, pp. 2809-2832, 2009.

[28] Y. H. Qian, J. Y. Liang, P. Song, and C. Y. Dang, "On dominance relations in disjunctive set-valued ordered information systems," International Journal of Information Technology \& Decision Making, vol. 9, no. 1, pp. 9-33, 2010.

[29] B. Huang, D.-K. Wei, H.-X. Li, and Y.-L. Zhuang, "Using a rough set model to extract rules in dominance-based interval-valued intuitionistic fuzzy information systems," Information Sciences, vol. 221, pp. 215-229, 2013.

[30] X. L. Zuo, W. J. Li, and L. Y. Cai, Discrete Mathematics, Shanghai Science and Technology Literature Press, Shanghai, China, 1982.

[31] A. Skowron, "Boolean reasoning for decision rules generation," in Methodologies for Intelligent Systems, vol. 689 of Lecture Notes in Computer Science, pp. 295-305, Springer, Berlin, Germany, 1993. 


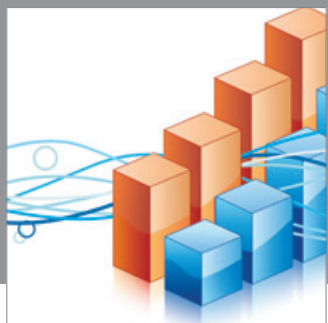

Advances in

Operations Research

mansans

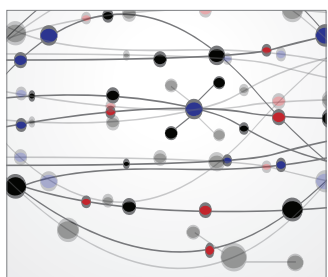

The Scientific World Journal
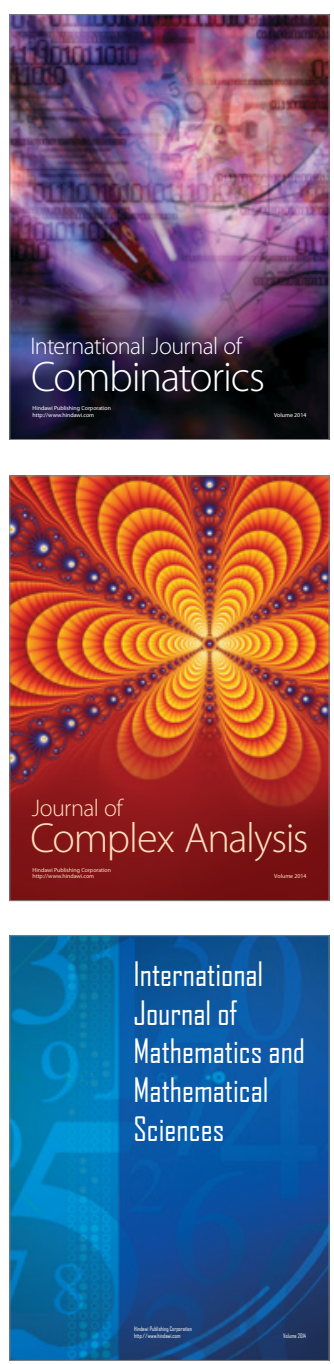
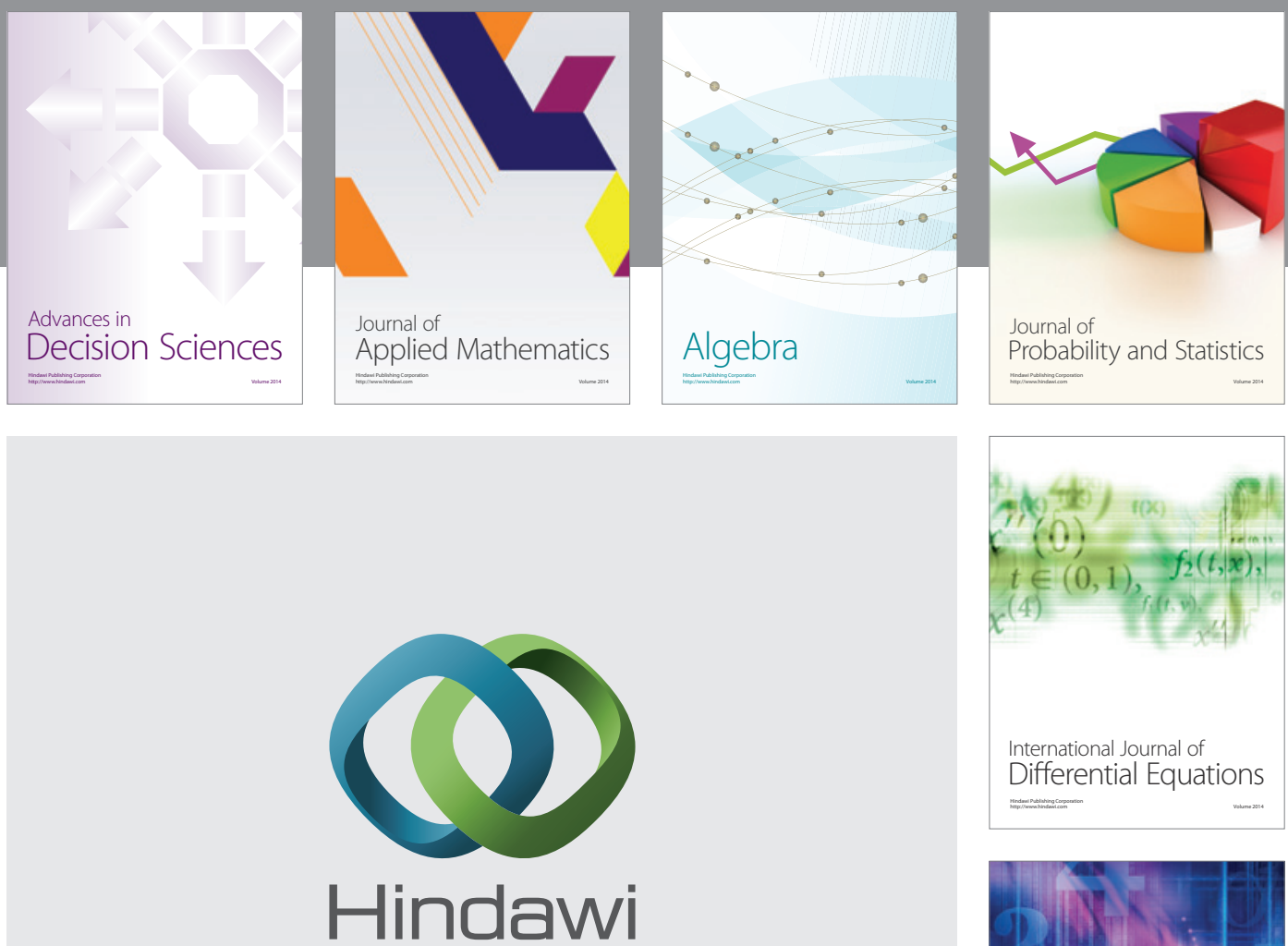

Submit your manuscripts at http://www.hindawi.com
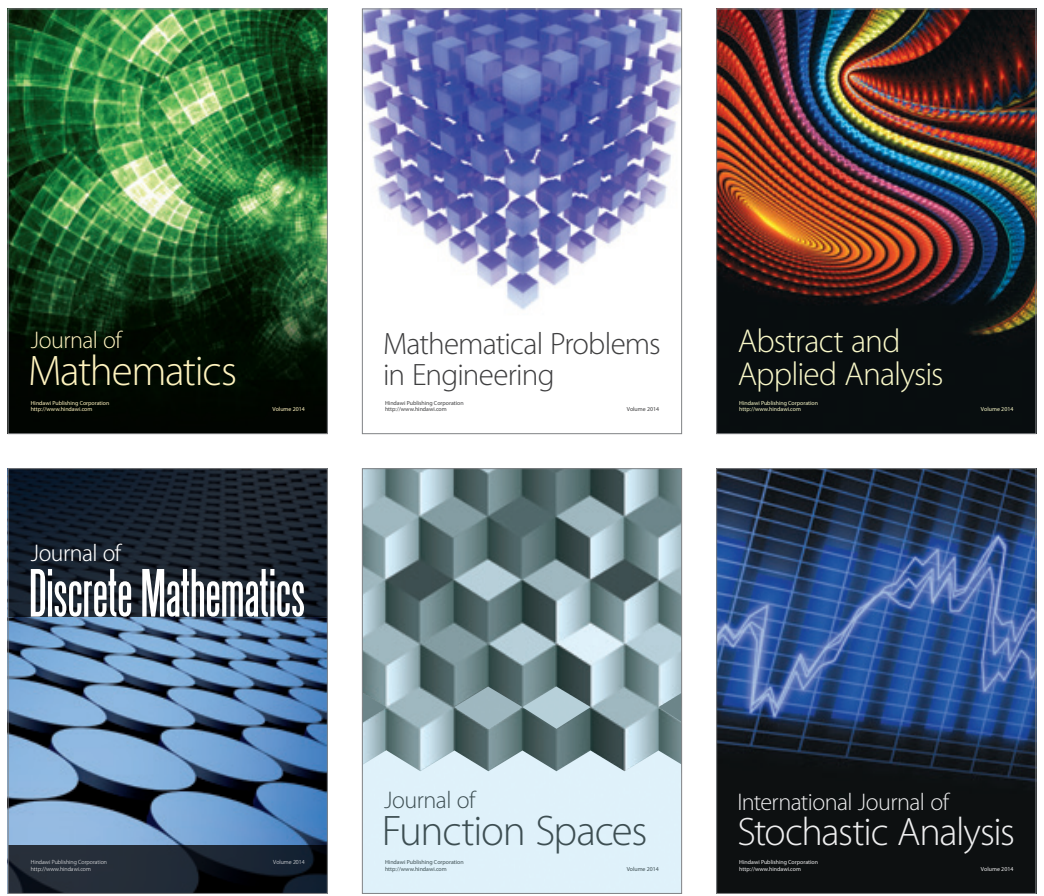

Journal of

Function Spaces

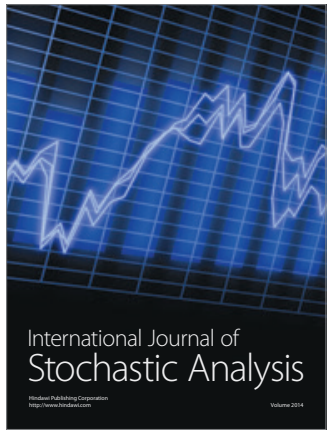

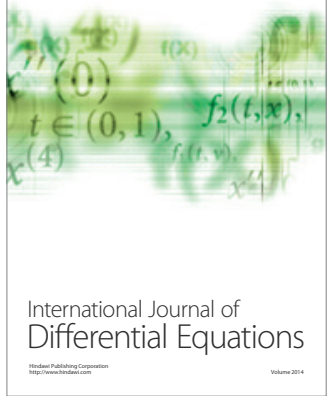
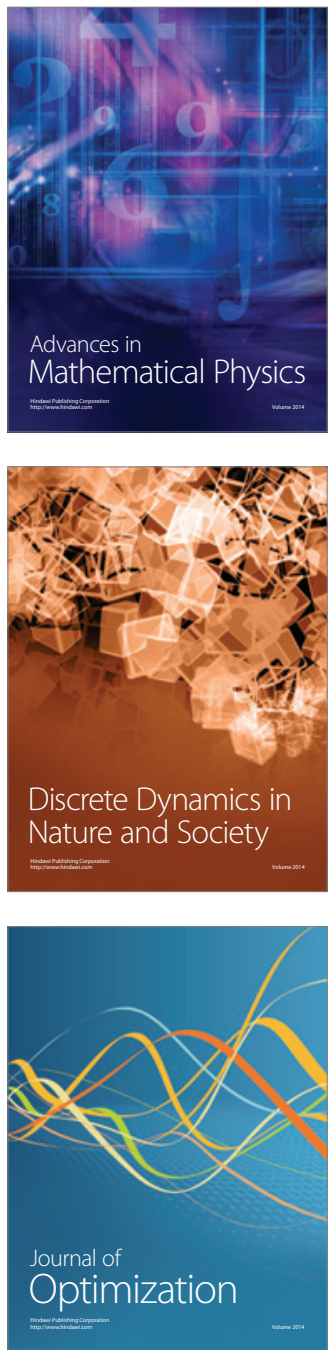